\title{
Insuficiencia pancreática exocrina por atrofia pancreática en un perro: informe de caso
}

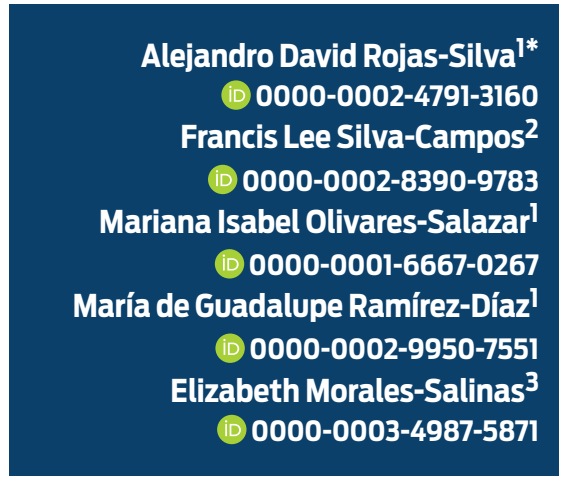

IUniversidad Nacional Autónoma de México, Facultad de Medicina Veterinaria y Zootecnia, Departamento de Patología, Área de Patología Clínica

${ }^{2}$ Facultad de Medicina Veterinaria y Zootecnia, Universidad Nacional Autónoma de México; Departamento de Medicina, Cirugía y Zootecnia de Perros y Gatos,

Hospital Veterinario de Especialidades en Pequeñas Especies.

${ }^{3}$ Facultad de Medicina Veterinaria y Zootecnia, Universidad Nacional Autónoma de México; Departamento de Patología. Área de Anatomopatología

* Autor para correspondencia: Correo electrónico: davidrojassilva@gmail.com

Recibido: 2018-12-19 Aceptado: 2019-07-30

Publicado: 2019-09-12

Información y declaraciones adicionales en la página 10

(a) Derechos de autor: Alejandro David Rojas-Silva et al. 2019

acceso abierto $\mathbf{O}$

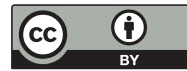

Distribuido bajo una Licencia Creative Commons Atribución 4.0 Internacional (CC-BY 4.0)

\section{Resumen}

Descripción del caso. Se presentó al Hospital Veterinario de Especialidades UNAM (HVE-UNAM) un perro, macho entero, de un año seis meses, bulldog francés, el motivo de consulta fue pérdida progresiva de peso, diarrea con cuatro meses de evolución, y uno a dos vómitos por día.

Hallazgos clínicos. Condición corporal (CC) 1/5. En la palpación transabdominal, asas intestinales engrosadas, derrame abdominal y posible cuerpo extraño observado mediante ultrasonido abdominal.

Tratamiento y evolución. Se le administró ranitidina, metronidazol, solución Hartmann, fenbendazol, omeprazol, citrato de maropitant, prednisona, nalbufina y alimento gastrointestinal bajo en grasa, sin respuesta favorable. Pruebas de laboratorio. Se realizaron hemogramas, bioquímicas séricas, gasometría, pruebas de función pancreática y estudio de necropsia. Los resultados fueron consistentes con insuficiencia pancreática exocrina secundaria a atrofia acinar pancreática.

Relevancia clínica. La insuficiencia pancreática exocrina (IPE) es un síndrome caracterizado por la síntesis y secreción inadecuada de enzimas digestivas pancreáticas debido a un daño severo del órgano o falta de células acinares. Una de las causas más comunes es la atrofia acinar pancreática (AAP) en pacientes jóvenes como consecuencia de un trastorno genético complejo. La mayoría de los casos no demuestran signos clínicos evidentes hasta los doce meses de edad, por lo que el diagnóstico en etapas tempranas y el pronóstico no suelen ser favorables. Es necesario realizar un buen examen

Una forma de citar este artículo:

Rojas-Silva AD, Silva-Campos FL, Olivares-Salazar MI, Ramírez-Díaz M, de G, MoralesSalinas E. Insuficiencia pancreática exocrina por atrofia pancreática en un perro: informe de caso. Clínica veterinaria: abordaje diagnóstico y terapéutico. 2019;5:e41201956. doi:10.22201/fmvz.23958766e.201941. 
físico, varios exámenes de laboratorio y de imagen, para llegar al diagnóstico definitivo. El estudio y descripción de esta enfermedad es necesario para entender de mejor manera la fisiopatología, que permita realizar el tratamiento oportuno y mejorar así la calidad de vida de estos pacientes.

Palabras clave: perro, diarrea, enfermedad pancreática exocrina, atrofia acinar pancreática.

\section{Exocrine insufficiency for atrophy pancreatics in a dog: a case report}

\section{Abstract}

Case description. A french bulldog male one year and six months old was presented at the Hospital Veterinario de Especialidades UNAM (HVE-UNAM); the dog owner reported that in the last four months presented diarrhea, progressive weight loss and one or two pukes per day.

Clinical findings. Body condition $1 / 5$, in transabdominal palpation it was perceived thickened loop of bowel, abdominal effusion and a possible foreign body observed by abdominal ultrasound.

Treatment and evolution. Ranitidine, metronidazole, Hartmann solution, fenbendazole, omeprazole, maropitant citrate, prednisone, nalbuphine and low-fat gastrointestinal food were given without favorable response.

Laboratory tests. Blood count cells, serum biochemistry profile, gasometry, pancreatic function tests and necropsy study were performed. The results were consistent with exocrine pancreatic insufficiency due to pancreatic acinar atrophy.

Clinical relevance. Exocrine pancreatic insufficiency is a syndrome characterized by inadequate synthesis and secretion of pancreatic digestive enzymes due to severe organ damage or lack of acinar cells. One of the most common causes is pancreatic acinar atrophy in young patients as a result of a complex genetic disorder. Most of the cases do not show obvious clinical signs until twelve months old, so early diagnosis and prognosis are usually not favorable. In order to perform a definitive diagnosis is necessary to do an exhaustive physical examination, several laboratory and imaging tests. Thorough knowledge and description about this disease are necessary to understand the pathophysiology to timely treatment and thus improve the quality of life of these patients.

Keywords: dog, diarrhea, exocrine pancreatic disease, pancreatic acinar atrophy. 


\section{Descripción del caso}

Se presentó el 16 de noviembre del 2017 (día 1) al Hospital Veterinario de Especialidades UNAM (HVE-UNAM) un perro, macho entero, de un año seis meses, bulldog francés, porque de manera progresiva perdió peso, tuvo diarrea con cuatro meses de evolución, y uno a dos vómitos por día. En septiembre del 2017, los propietarios empezaron a ofrecer alimento húmedo y proteínas hidrolizadas, y en octubre, se inició tratamiento con prednisona y omeprazol por indicaciones de otro médico.

\section{Hallazgos clínicos e interpretación}

En el examen físico general (EFG) del día 1, se observó una condición corporal (CC) 1/5 y en la palpación transabdominal, asas intestinales engrosadas. Los diagnósticos presuntivos fueron enteritis secundaria a cuerpo extraño, o linfoma alimentario. Se realizó estudio radiográfico de abdomen en el cual no se observaron anormalidades.

En la ecografía abdominal y torácica, se observó derrame y estructuras que generaban sombra acústica en estómago sugerente de cuerpo extraño. La medición de albúmina sérica estaba dentro de intervalos de referencia. Se dio de alta hospitalaria.

El día 30, el paciente acudió nuevamente por vómito, diarrea pastosa y pérdida de peso, se realizó ultrasonido en donde se observó un cuerpo extraño de $1.72 \mathrm{~cm}$ en su eje mayor en estómago, no generaba obstrucción, aumento del grosor en la pared del duodeno, yeyuno e íleon, puntilleo hiperecoico en mucosa sugerente de enfermedad inflamatoria intestinal (EII). El paciente se quedó hospitalizado y se inició el tratamiento por la probable Ell. Se enviaron muestras de heces para estudio coproparasitoscópico seriado que resultó negativo. Se realizaron hemograma y bioquímica sérica (Cuadros 1 y 2).

El día 32 no se observaron cambios clínicos relevantes, se tomó muestra para perfil pancreático exocrino. Los días 33 y 34, el paciente presentó mala evolución clínica. Se propuso realizar biopsia intestinal para confirmar diagnóstico de Ell.

Los días 35 y 36, presentó diarrea y dolor abdominal, debido a la pobre respuesta al tratamiento y la mala evolución clínica, se ingresó al área de terapia intensiva en donde presentó estupor. Se solicitó hemograma, bioquímica sérica (Cuadros 1 y 2) y gasometría venosa. Debido a los resultados del hemograma se realizó transfusión sanguínea; sin embargo, por la mala evolución clínica del paciente, se decidió aplicar la eutanasia.

\section{Tratamiento}

Se le administró ranitidina a 2 mg/kg PO BID durante 21 días, metronidazol a 15 mg/ kg PO BID 21 días, solución Hartmann con la dosis de mantenimiento más 8 \% de deshidratación (DH), fenbendazol a $50 \mathrm{mg} / \mathrm{kg}$ PO SID durante cinco días, omeprazol a $1 \mathrm{mg} / \mathrm{kg}$ IV SID, sucralfato $500 \mathrm{mg} / \mathrm{kg}$ PO TID, citrato de maropitant a $1 \mathrm{mg} /$ kg IV SID, prednisona a $2 \mathrm{mg} / \mathrm{kg}$ PO SID, nalbufina a $0.3 \mathrm{mg} / \mathrm{kg}$ IV TID, ampicilina a $22 \mathrm{mg} / \mathrm{kg}$ IV TID y alimento gastrointestinal bajo en grasa, sin respuesta favorable. 
Cuadro 1. Hemogramas

\begin{tabular}{|l|c|c|c|}
\hline \multicolumn{1}{|c|}{ Analito } & Día 30 & Día 35 & Referencia \\
\hline Hematocrito & 0.52 & 0.12 & $0.37-0.55 \mathrm{~L} / \mathrm{L}$ \\
\hline Eritrocitos & 8.4 & 2.1 & $(5.5-8.5) 10^{12} / \mathrm{L}$ \\
\hline Hemoglobina & 179 & 49 & $120-180 \mathrm{~g} / \mathrm{L}$ \\
\hline Reticulocitos & - & 32 & $(<60) 10^{9} / \mathrm{L}$ \\
\hline Plaquetas & 264 & - & $(148-484) 10^{9} / \mathrm{L}$ \\
\hline Neutrófillos & 11.5 & - & $(3-11.5) 10^{9} / \mathrm{L}$ \\
\hline Linfocitos & 0.4 & - & $(1-4.8) 10^{9} / \mathrm{L}$ \\
\hline Eosinófílos & 0 & - & $(0.06-1.23) 10^{9} / \mathrm{L}$ \\
\hline
\end{tabular}

Cuadro 2. Bioquímicas séricas

\begin{tabular}{|l|c|c|c|}
\hline \multicolumn{1}{|c|}{ Analito } & Día 30 & Día 35 & Referencia \\
\hline Clucosa & 6.4 & 3.97 & $3.88-6.88 \mathrm{mmol} / \mathrm{L}$ \\
\hline Urea & 17.4 & 19.5 & $2.1-7.9 \mathrm{mmol} / \mathrm{L}$ \\
\hline Triglicéridos & 0.5 & 0.4 & $0.6-1.2 \mathrm{mmol} / \mathrm{L}$ \\
\hline Colesterol & 0.75 & 0.7 & $2.84-8.26 \mathrm{mmol} / \mathrm{L}$ \\
\hline ALT & 275 & 232 & $>70 \mathrm{U} / \mathrm{L}$ \\
\hline AST & 88 & - & $>55 \mathrm{U} / \mathrm{L}$ \\
\hline CK & 519 & - & $>213 \mathrm{U} / \mathrm{L}$ \\
\hline Proteinas totales & 54 & 38 & $56-75 \mathrm{~g} / \mathrm{L}$ \\
\hline Globulinas & 22 & 24 & $23-39 \mathrm{~g} / \mathrm{L}$ \\
\hline Albuimina & 32 & 14 & $23-40 \mathrm{~g} / \mathrm{L}$ \\
\hline Relación A/G & 0.68 & 1.71 & $0.78-1.46$ \\
\hline
\end{tabular}

\section{Pruebas de laboratorio}

El día 30, en el hemograma se observó linfopenia de $0.4 \times 10^{9} / \mathrm{L}\left(1-4.8 \times 10^{9} / \mathrm{L}\right)$ por acción de glucocorticoides exógenos (tratamiento con prednisona), mientras que en la bioquímica sérica las alteraciones fueron: hiperuremia de $17.4 \mathrm{mmol} / \mathrm{L}$ (2.7-7.9 mmol/L) que se interpreta como hiperazotemia prerrenal por hemoconcentración, hipotrigliceridemia de $0.5 \mathrm{mmol} / \mathrm{L}(0.6-1.2 \mathrm{mmol} / \mathrm{L})$, hipocolesterolemia de $0.75 \mathrm{mmol} / \mathrm{L}(2.84-8.36 \mathrm{mmol} / \mathrm{L})$ e hipoproteinemia de $54 \mathrm{~g} / \mathrm{L}$ (56-75 g/L) por pérdidas entéricas y disminución en su absorción, incremento de ALT de $275 \mathrm{U} / \mathrm{L}(<70 \mathrm{U} / \mathrm{L})$ y AST de $88 \mathrm{U} / \mathrm{L}(<55 \mathrm{U} / \mathrm{L})$ por daño hepatocelular activo, aumento de $\mathrm{CK}$ de $519 \mathrm{U} / \mathrm{L}(<213 \mathrm{U} / \mathrm{L})$ y parte del incremento de AST por esfuerzo muscular.

El día 32, se realizaron pruebas de función pancreática, los resultados fueron tripsina inmunoreactiva de $3.2 \mu \mathrm{g} / \mathrm{L}(5-35 \mu \mathrm{g} / \mathrm{L})$, cobalamina en $150 \mathrm{ng} / \mathrm{L}$ (284-836 ng/L) y folato de $19.6 \mu \mathrm{g} / \mathrm{L}(4.8-19 \mu \mathrm{g} / \mathrm{L})$ compatible con insuficiencia pancreática exocrina.

El día 35, en el hemograma se presentó hematocrito de $0.12 \mathrm{~L} / \mathrm{L}(0.37-0.55 \mathrm{~L} / \mathrm{L})$ y reticulocitos en $32 \times 10^{9} / \mathrm{L}\left(<60 \times 10^{9} / \mathrm{L}\right)$ correspondiente con anemia severa no regenerativa y monocitosis de $1.8 \times 10^{9} / \mathrm{L}\left((0.1-1.4) \times 10^{9} / \mathrm{L}\right)$ por inflamación 
crónica y parte de la anemia por posible disminución en la absorción de hierro a nivel intestinal, mientras que en la bioquímica sérica observamos hiperuremia de $19.5 \mathrm{mmol} / \mathrm{L}$ (2.7-7.9 mmol/L) que interpretamos como hiperazotemia prerrenal por hemoconcentración, hipotrigliceridemia de $0.4 \mathrm{mmol} / \mathrm{L}(0.6-1.2 \mathrm{mmol} / \mathrm{L})$, hipocolesterolemia de $0.7 \mathrm{mmol} / \mathrm{L}(2.84-8.26 \mathrm{mmol} / \mathrm{L})$ e hipoproteinemia de $38 \mathrm{~g} / \mathrm{L}(56-75 \mathrm{~g} / \mathrm{L}$ ) por disminución en su absorción y pérdidas entéricas, además, incremento de ALT de $232 \mathrm{U} / \mathrm{L}(<70 \mathrm{U} / \mathrm{L})$ por daño hepatocelular.

El mismo día se realizó gasometría venosa en la que se observó hipobicarbonatemia de $17.5 \mathrm{mmol} / \mathrm{L}$ (18-24 mmol/L) e hipercloremia de $121 \mathrm{mmol} / \mathrm{L}$ (108$117 \mathrm{mmol} / \mathrm{L}$ ) por acidosis metabólica hiperclorémica, hipocaliemia de $3.3 \mathrm{mmol} / \mathrm{L}$ (3.5-5.5 mmol/L) por pérdidas intestinales, hipocalcemia $\left(\mathrm{Ca}^{2}+\mathrm{i}\right)$ de $0.99 \mathrm{mmol} / \mathrm{L}$ (1.2-1.5 mmol/L) e hipoglucemia marcada de $0.9 \mathrm{mmol} / \mathrm{L}(3.8-6.8 \mathrm{mmol} / \mathrm{L})$ por disminución en su absorción.

El día 37, se realizó la necropsia donde el examen macroscópico informó condición corporal 1/5 con marcada pérdida de masa muscular (Figura 1) y de tejido adiposo subcutáneo, $3 \mathrm{~mL}$ de líquido (trasudado simple) en saco pericárdico y degeneración mucoide de la grasa pericárdica. Lo más relevante fue la marcada disminución de tamaño y la pérdida de la arquitectura normal del páncreas (atrofia) (Figura 2).

En el estómago se encontró un fragmento de $4.5 \times 2.5 \mathrm{~cm}$ de un cuerpo extraño (cinturón de cuero). En el estudio microscópico del páncreas se informó la pérdida de la arquitectura normal del órgano, así como la pérdida de la afinidad tintorial. Células acinares con escaso citoplasma de bordes poco distinguibles, que no se diferenciaban de los islotes de Langerhans. En algunas zonas se observaron pequeños acinos y se apreciaron áreas con conductos pancreáticos (Figura 3).

Con todos los hallazgos, se diagnosticó atrofia pancreática, alteración congénita poco frecuente, que provoca mala digestión por ausencia en la producción de enzimas pancreáticas y pérdida de peso debido a la digestión deficiente, lo que provoca poca absorción de nutrientes, diarrea y emaciación progresiva del paciente.

\section{Discusión y relevancia clínica}

La IPE es un síndrome caracterizado por la síntesis y secreción inadecuada de enzimas digestivas pancreáticas debido al daño severo o a la falta de células acinares pancreáticas. Las causas más comunes son la atrofia acinar pancreática (AAP) en pacientes jóvenes, ${ }^{1}$ es más frecuente en el pastor alemán, el collie y las razas asiáticas; otra causa es la pancreatitis crónica, que resulta de la destrucción de las células acinares que inicialmente se relacionó con un patrón de herencia autosómico recesivo, estudios recientes sugieren que es consecuencia de un trastorno genético más complejo y que probablemente involucre múltiples genes (incluidos los alelos del complejo mayor de histocompatibilidad); factores ambientales como la alimentación, el estrés y algunos virus. ${ }^{2}$ Otras alteraciones menos comunes son neoplasias, aplasia congénita e hipoplasia pancreática.

La mayoría de los casos no muestran signos clínicos hasta cumplir los 6-12 meses de edad. ${ }^{3}$ En este caso, el perro era un bulldog frances de un año seis meses, la literatura no reporta esta raza como predisponente a IPE, sin embargo 


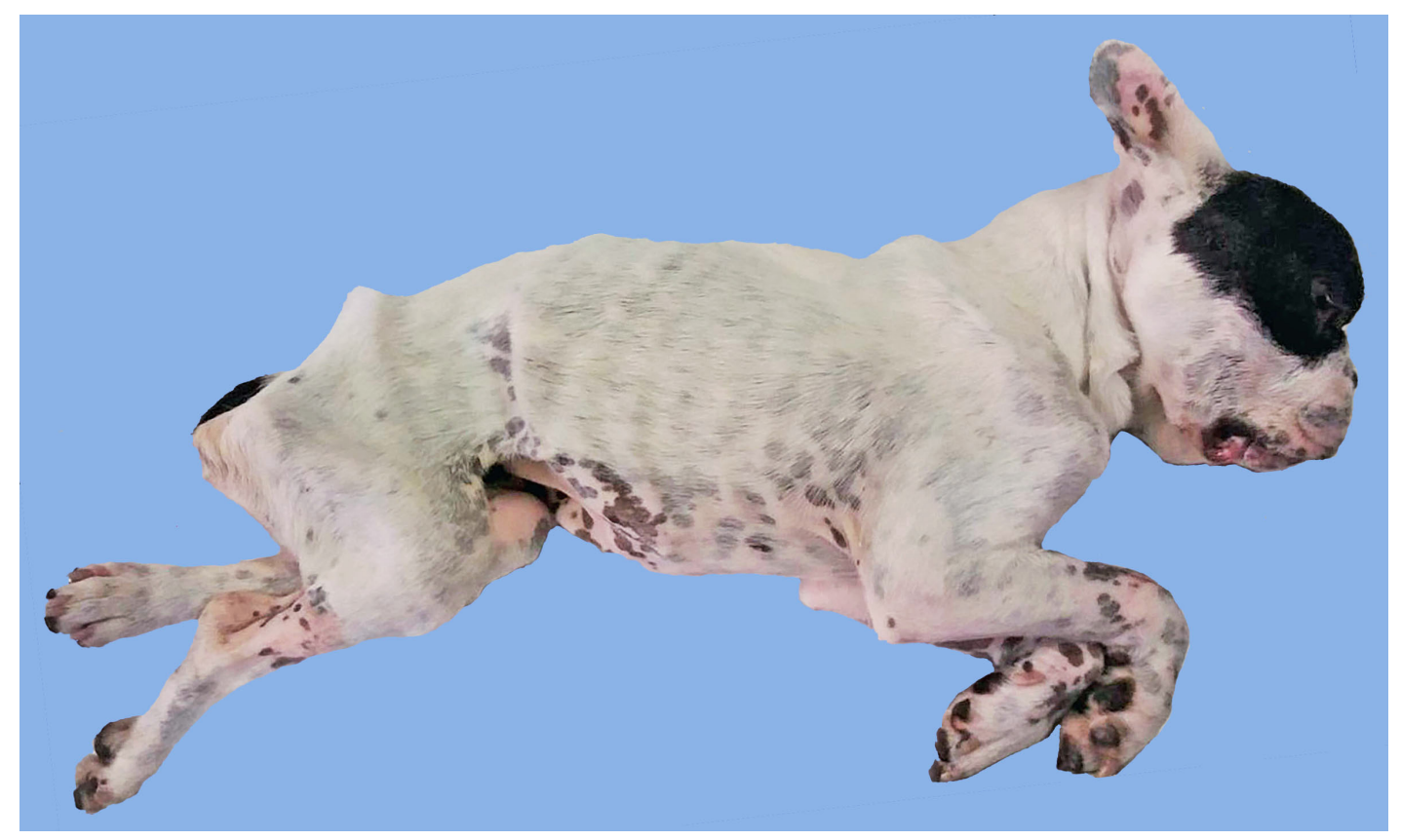

Figura 1. Perro, bulldog francés. Se aprecia la baja condición corporal del cuerpo en la necropsia (CC 1/5).

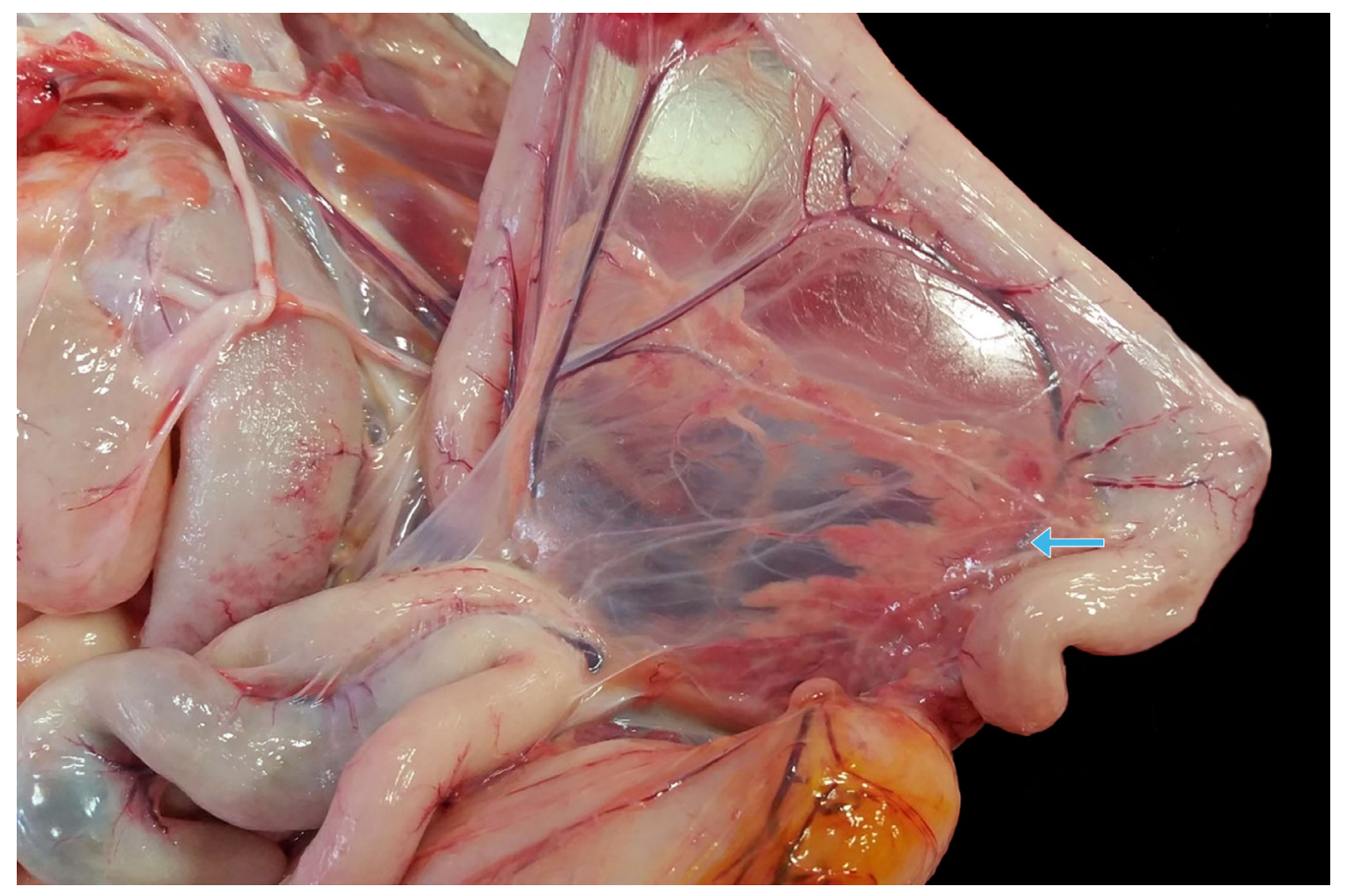

Figura 2. Intestino y páncreas. Disminución marcada del tejido pancreático (flecha azul). 


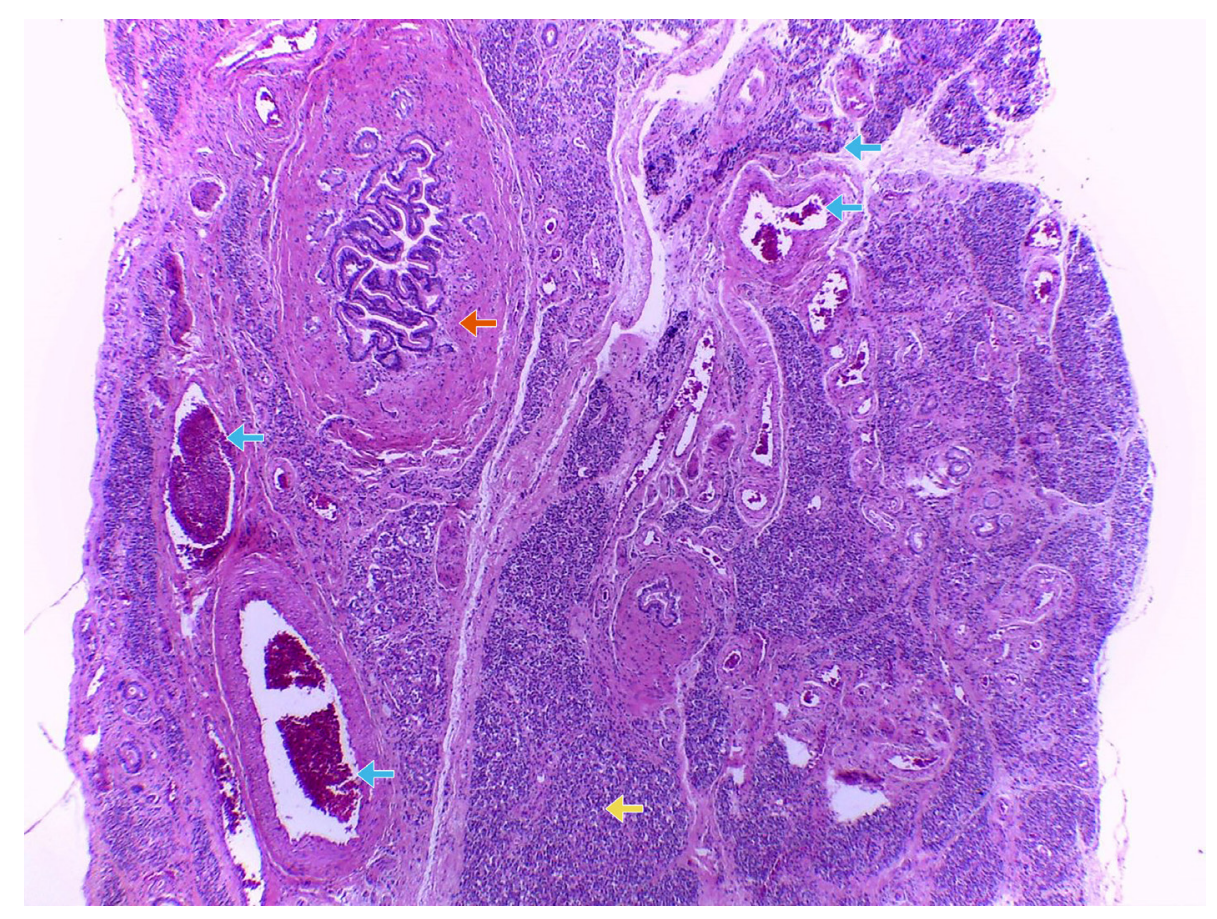

Figura 3. Corte histológico del páncreas, H\&E, 4X. Se aprecia desarreglo de la arquitectura del parénquima, los vasos sanguíneos están dilatados y congestionados (flechas azules), y el tejido que rodea a los ductos se aprecia engrosado por tejido conectivo (flecha naranja), de estos se deriva gran cantidad de estrías de tejido conectivo que disecan los acinos, donde el tejido tiene una alta celularidad sin el patrón acinar característico (flecha amarilla).

si se ha reportado en otras razas. De acuerdo con la edad del paciente, los signos clínicos y hallazgos en la necropasia, la causa de la IPE fue AAP juvenil. La aparición de los signos clínicos en este paciente concuerda con lo indicado en la literatura.

Una vez instaurada la IPE, se producen alteraciones nutricionales debido a la falta de enzimas pancreáticas y a la disminución en la absorción del intestino. ${ }^{1}$ En las primeras etapas de la atrofia del parénquima exocrino, en la histopatología, se observa infiltración intensa pero irregular de los linfocitos T denominada pancreatitis linfocítica atrófica. Donde los linfocitos citotóxicos CD8 predominan sobre los linfocitos CD4. A medida que la enfermedad progresa, los linfocitos se pueden encontrar alrededor de las células acinares, así como en el epitelio ductal; por lo que la lesión sugiere que la condición puede ser un proceso mediado por células autoinmunes dirigidas contra células acinares; sin embargo, no hay evidencia de la participación de autoanticuerpos en la patogenia de esta enfermedad. ${ }^{3-4}$

En los hallazgos microscópicos de la necropsia en páncreas, observamos infiltración linfocítica, característica de la AAP (Figuras 4 y 5). Las pruebas de laboratorio de rutina fueron hemograma, bioquímica sérica y urianálisis, importantes para dirigir el diagnóstico de IPE; a pesar de ello, es necesario realizar pruebas específicas para llegar al diagnóstico certero. ${ }^{2}$

Dentro de los analitos de laboratorio para el diagnóstico de IPE en perros, se menciona la medición de cobalamina, folato y la prueba de tripsina inmunorreactiva (TLI). Las dos primeras son poco eficientes para el diagnóstico debido a que pueden estar alteradas por disbiosis intestinal como se produce en el síndrome de malabsorción por Ell. ${ }^{2}$ La concentración sérica de TLI es la prueba con mayor valor 


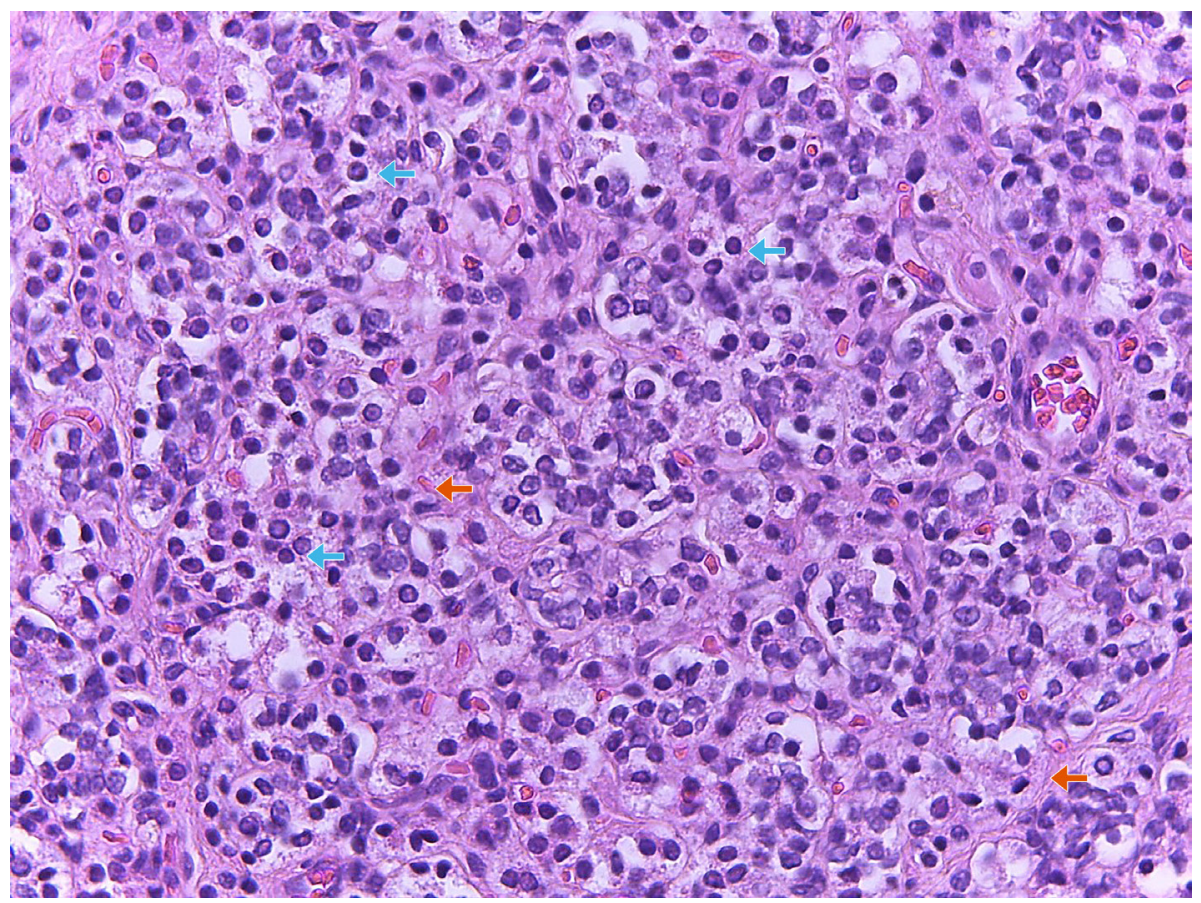

Figura 4. Corte histológico del páncreas, H\&E, 40X: sección de parénquima pancreático donde se aprecia abundante infiltrado inflamatorio mononuclear, principalmente de linfocíticos (flechas azules), con moderada a severa degeneración de las células acinares, con moderada presencia de tejido conectivo fibroso en el parénquima (flechas naranjas).

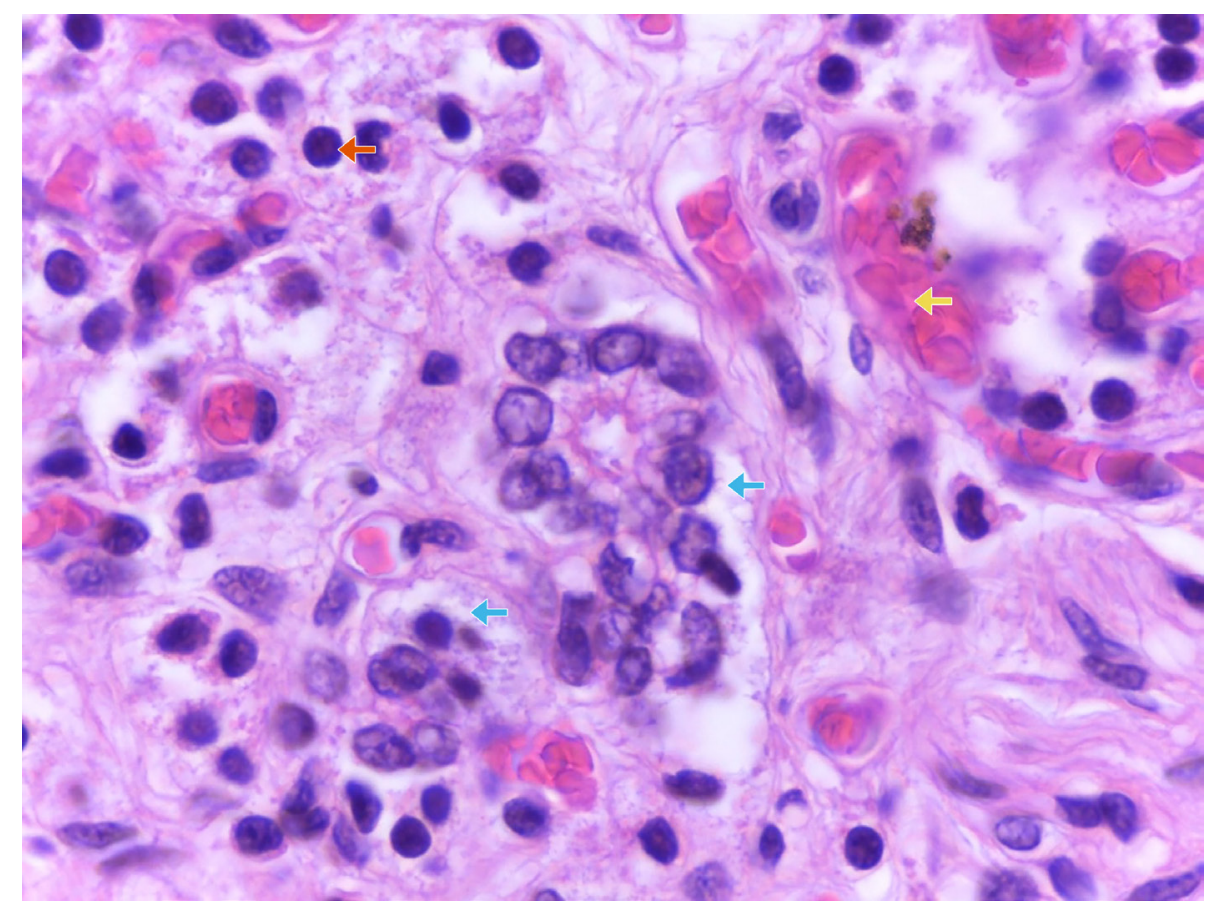

Figura 5. Corte histológico del páncreas, H\&E, 100X. Acinos donde se aprecia vacuolización en el citoplasma de las células acinares (flecha azul), células inflamatorias mononucleares, principalmente linfocitos (flecha naranja) y células plasmáticas sobre éstas y en el intersticio. Congestión moderada y microhemorragias discretas multifocales (flecha amarilla). 
diagnóstico para IPE en perros, en ocasiones los resultados de esta prueba pueden ser confusos en aquellos animales que no presentan signos clínicos; por ejemplo, en un estudio en el que se dio seguimiento a 71 perros enfermos, y al determinar $\mathrm{TLI}$, el $50 \%$ de la población presentó un valor por debajo del límite inferior de referencia $\left(5 \mu \mathrm{g} / \mathrm{L}_{l}\right)$ pero sin signos clínicos de la enfermedad. ${ }^{5-6}$

Otras pruebas complementarias para evaluar la función digestiva en IPE son la medición de grasa fecal (esteatorrea), proteína fecal (creatorrea) y almidón en heces (amilorrea), pero ninguna tiene una alta especificidad ni sensibilidad, pues se alteran por enfermedad inflamatoria intestinal como en la esteatorrea, por el sobrecrecimiento bacteriano o la enfermedad celiaca.

En medicina humana, la prueba más utilizada para evaluar IPE es la elastasa fecal, con $100 \%$ de sensibilidad en IPE severa, 77-100 \% cuando es moderada y 0-63\% en casos leves. La especificidad es del $93 \%$ aunque puede alterarse en pacientes con enfermedad del intestino delgado o con diabetes insulino dependientes. ${ }^{7-8}$

En este bulldog francés, no se evaluó la función digestiva por su poca eficacia para detectar o confirmar IPE, y tampoco la medición de elastasa fecal ya que en medicina veterinaria la medición de TLI es la prueba de oro para IPE. En el momento en que surgen los signos clínicos, la atrofia exocrina está avanzada y por lo general queda poca evidencia de los eventos degenerativos e inflamatorios precedentes. Debido a los resultados en las pruebas de laboratorio (hemograma, bioquímica sérica, medición de cobalamina, folato y TLI) en conjunto con los signos clínicos, la historia clínica y la anamnesis del animal de este caso clínico, el diagnóstico fue de IPE y solamente con la necropsia se pudo determinar la AAP.

En la necropsia de perros clínicamente afectados, los intestinos se observan distendidos o inflamados por ingesta voluminosa o el consumo de objetos extraños. ${ }^{3}$ Histológicamente, hay disminución marcada de células acinares pancreáticas exocrinas y, a veces, se ven afectadas células de los islotes (páncreas endocrino).2,4 En este caso, el perro no mostró hallazgos histológicos sugerentes de daño a la porción endocrina del páncreas.

El tratamiento para la IPE consiste en la administración oral de enzimas pancreáticas. Todo paciente con IPE, independientemente del grado de esteatorrea y presencia o ausencia de signos clínicos, debería recibir la terapia de remplazo enzimático pancreático (TREP). El mejor parámetro para evaluar el tratamiento es la ganancia de peso corporal. Las enzimas utilizadas en la TREP son de origen porcino.

En algunos casos (creencias religiosas) se pueden utilizar enzimas de origen bovino, aunque poseen 75\% menos actividad en la lipasa, comparadas con las de origen porcino. Otras alternativas, aun en estudio, son las lipasas de origen micótico, bacteriano y humano. La dosis recomendada en la TREP es de 25000 a 75000 unidades de lipasa por comida y de 10000 a 25000 unidades por bocadillo, esto dependerá de la cantidad de grasa de cada comida.

La extensión del tejido pancreático y la respuesta al TREP determinan la sobrevida del paciente entre uno y cinco años. ${ }^{9}$ Se platicó con el propietario del paciente sobre la TREP, más se evitó por falta de recursos económicos. Como tratamiento complementario se indica dieta baja en grasa con alto contenido de proteínas y fibra, antibióticos (oxitetraciclina, amoxicilina con ácido clavulánico, enrofloxacina, sulfonamidas y tilosin, entre los utilizados) para reducir la cantidad de bacterias en 
el intestino y las posibles infecciones secundarias, antagonistas de receptores $\mathrm{H}_{2}$ y suplemento con cobalamina. ${ }^{9}$

El paciente descrito recibió antibioterapia y antagonistas de $\mathrm{H}_{2}$, sin la TREP como terapia principal, no se observó mejoría clínica. Para llegar a determinar IPE secundaria a atrofia pancreática, se requiere de una anamnesis adecuada, la evaluación clínica completa del paciente, el perfil integral de rutina (hemograma, bioquímica y urianálisis) y estudios de imagenología para encaminar el diagnóstico; sin embargo, las pruebas complementarias como la medición de TLI, cobalamina y folato pueden ser de mayor utilidad para el diagnóstico definitivo.

Una vez dirigido el diagnóstico de IPE, la biopsia incisional de páncreas, podría ayudar a determinar el origen de la enfermedad. En este perro, los signos clínicos que manifestó estaban asociados a múltiples patologías como pancreatitis, IPE, Ell y gastroenteritis de diversos orígenes, por lo que la atrofia pancreática se pudo diagnosticar mediante la necropsia y se correlacionó con los hallazgos clínicos y las pruebas de laboratorio.

\section{Financiamiento}

Las pruebas realizadas fueron costeadas por el propietario del paciente..

\section{Conflictos de interés}

Los autores declaran que no hay conflicto de intereses.

\section{Contribución de los autores}

ADRS, redacción del artículo, ejecución e interpretación de las pruebas de laboratorio en el área de patología clínica.

FLSC, abordaje, seguimiento y tratamiento clínico.

MOS, procesamiento e interpretación de las pruebas de histopatología.

MGRD, supervisión y revisión del artículo.

EMS, supervisión y revisión del artículo.

\section{Referencias}

1. Cyrus J, Steiner, J. Exocrine pancreatic insufficiency in dogs. NAVC Clinician's Brief [internet]. 2011 [citado 10 junio 2018]:55-9. Disponible en: https://www. cliniciansbrief.com/article/exocrine-pancreatic-insufficiency-dogs

2. Westermarck E, Wiberg M. Exocrine pancreatic insufficiency in the dog: historical background, diagnosis, and treatment. Top Companion Anim Med [internet]. 2012 [citado 13 junio 2018]; 27:96-103. Disponible en: https://www.ncbi. nlm.nih.gov/pubmed/23148848

3. Kenneth VF, Jubb, Stent AW. Chapter 3 - Pancreas. In: M. Grant Maxie, editor. Jubb, Kennedy \& Palmer's pathology of domestic animals: Guelph, Ontario-(CA): Elsevier; 2015. p. 353-75.e1. doi: 10.1016/B978-0-7020-5318-4.00009-7.

4. Guarín-Patarroyo C, Sánchez C. Insuficiencia pancreática exocrina (IPE) canina. Revista Logos. Ciencia y Tecnología [internet]. 2013 [citado 13 junio 2018];5(1): 84-96. Disponible en: http://www.redalyc.org/pdf/5177/517751547008.pdf

5. Simpson K. Función de la nutrición en la patogenia y manejo de las alteraciones del páncreas exocrino. Enciclopedia de la clínica canina [Internet]. 2010 
[citado 15 junio 2018]: 173-198. Disponible en: http://www.ivis.org/advances/ rc_es/A4305.0208.ES.pdf?LA=2

6. Mansfield C. Practical interpretation and application of exocrine pancreatic testing in small animals. Vet Clin Small Anim [internet]. 2013 [citado 13 junio 2018]:1241-60. Disponible en: https://www.ncbi.nlm.nih.gov/ pubmed/24144088

7. Sikkens EC, Cahen DL, Kuipers EJ, Bruno MJ. Pancreatic enzyme replacement therapy in chronic pancreatitis. Best Practice \& Research Clinical Gastroenterology [internet]. 2010 [citado 19 junio 2019]:337-47. Disponible en: https://www. ncbi.nlm.nih.gov/pubmed/20510833

8. García PP, López G. Study on intestinal absorption, metabolism, and adaption. Nutr. Hosp. [internet]. 2007 [citado 19 junio 2019]:5-13. Disponible en: http://scielo. isciii.es/scielo.php?script=sci_abstract\&pid=S0212-161 12007000500002\&ln$\mathrm{g}=\mathrm{es} \& \mathrm{nrm}=\mathrm{iso} \& \operatorname{lng}=\mathrm{en}$

9. Batchelor DJ, Noble PJ, Taylor RH, Cripps PJ, German AJ. Pronostic factors in canine exocrine pancreatic insufficiency: prolonged survival is likely if clinical remission is achived. J Vet Inter Med. [internet]. 2007 [citado 19 junio 2019]:5460. Disponible: https://www.ncbi.nlm.nih.gov/pubmed/17338150 